\title{
Correction to: An efficient protocol for the synthesis of highly sensitive indole imines utilizing green chemistry: optimization of reaction conditions
}

Bushra Nisar ${ }^{1}$. Syeda Laila Rubab ${ }^{2}$. Abdul Rauf Raza ${ }^{3}$. Sobia Tariq ${ }^{3}$. Sana Jamshaid ${ }^{3}$. Ayesha Sultan ${ }^{4}$. Muhammad Nawaz Tahir ${ }^{5}$

Published online: 16 October 2018

(c) Springer Nature Switzerland AG 2018

\section{Correction to:}

Molecular Diversity (2018) 22:709-722

https://doi.org/10.1007/s11030-018-9826-3

In the original publication, one of the co-authors name Sana Jamshaid was missed out. The correct authors' group is updated in this correction.

The original article can be found online at https://doi.org/10.1007/s11 030-018-9826-3.

Abdul Rauf Raza

rauf.raza@uos.edu.pk

Sana Jamshaid

sanajamshaid41@gmail.com

1 Department of Chemistry, The University of Lahore,

Sargodha Campus, Lahore Road, Sargodha 40100, Pakistan

2 Department of Chemistry, University of Education, Jauharabad Campus, Jauharabad 41200, Pakistan

3 Ibn e Sina Block, Department of Chemistry, University of Sargodha, Sargodha 40100, Pakistan

4 Department of Chemistry, University of Education, Faisalabad Campus, Faisalabad 38000, Pakistan

5 Ibn ul Haithum Block, Department of Physics, University of Sargodha, Sargodha 40100, Pakistan 